\title{
Simulation of risk of tuberculosis infection in healthcare workers in hospitals of an intermediate incidence country
}

\author{
J. OCHOA ${ }^{1 *}$, D. HINCAPIÉ-PALACIO ${ }^{1}$, H. SEPÚLVEDA ${ }^{1,2}$, D. RUIZ ${ }^{3,4}$, A. MOLINA ${ }^{3}$, \\ S. ECHEVERRI ${ }^{5,6}$, A. L. LEÓN ${ }^{6}$, A. R. ESCOMBE ${ }^{7}$ AND M. P. ARBELÁEZ ${ }^{1}$ \\ ${ }^{1}$ National School of Public Health, 'Héctor Abad Gómez', Epidemiology Group, University of Antioquia, \\ Medellín, Colombia \\ ${ }^{2}$ National School of Public Health, 'Héctor Abad Gómez', Occupational Health Group, University of Antioquia, \\ Medellín, Colombia \\ ${ }^{3}$ Department of Environmental Engineering, School of Engineering in Antioquia, Colombia. \\ ${ }^{4}$ International Research Institute for Climate and Society, Columbia University in the City of New York, USA \\ ${ }^{5}$ ESEMetrosalud, Medellín, Colombia \\ ${ }^{6}$ School of Medicine, University of Antioquia, Medellin, Colombia \\ ${ }^{7}$ Imperial College, Department of Infectious Diseases \& Immunity, London, UK
}

Received 11 September 2014; Final revision 24 November 2014; Accepted 25 November 2014; first published online 29 December 2014

\section{SUMMARY}

We simulated the frequency of tuberculosis infection in healthcare workers in order to classify the risk of TB transmission for nine hospitals in Medellín, Colombia. We used a risk assessment approach to estimate the average number of infections in three risk groups of a cohort of 1082 workers exposed to potentially infectious patients over 10- and 20-day periods. The risk level of the hospitals was classified according to TB prevalence: two of the hospitals were ranked as being of very high priority, six as high priority and one as low priority. Consistent results were obtained when the simulation was validated in two hospitals by studying 408 healthcare workers using interferon gamma release assays and tuberculin skin testing. The latent infection prevalence using laboratory tests was $41 \%$ [95\% confidence interval (CI) $34 \cdot 3-47 \cdot 7]$ and $44 \%(95 \%$ CI $36 \cdot 4-51 \cdot 0)$ in those hospitals, and in the simulation, it was $40 \cdot 7 \%$ (95\% CI $32 \cdot 3-49 \cdot 0)$ and $36 \%(95 \%$ CI 27.9-44.0), respectively. Simulation of risk may be useful as a tool to classify local and regional hospitals according to their risk of nosocomial TB transmission, and to facilitate the design of hospital infection control plans.

Key words: Latent tuberculosis infection, occupational exposure, risk assessment.

\section{INTRODUCTION}

Tuberculosis (TB) control plans for hospitals and healthcare workers (HCWs) are an important and often neglected component of control programmes

\footnotetext{
* Author for correspondence: Dr J. Ochoa, Facultad Nacional de Salud Pública 'Héctor Abad Gómez', Universidad de Antioquia, Calle 62 No. 52-59, Medellín, Colombia.

(Email: jesus.ochoa@udea.edu.co)
}

[1], especially in high and intermediate TB incidence countries where HCWs are highly exposed and infected with both drug-sensitive and drug-resistant strains of Mycobacterium tuberculosis [2]. Several studies show that HCWs have an increased frequency of latent tuberculosis infection (LTBI) and of TB illness compared to the general population [3, 4], and that basic and validated infection control measures are required to support infection control programmes in those countries [5]. 
Transmission of M. tuberculosis is more likely to occur in hospitals that do not promptly identify cases of pulmonary TB and tend to delay diagnosis, isolation and treatment. TB transmission also depends on variables not directly observable by infection control programmes such as exposure to the respiratory droplets produced by patients with pulmonary TB [6], and many other factors including the viability of $M$. tuberculosis in those droplets, and the ventilation of health facilities [6].

Simulation helps to understand the relationship between these stochastic variables and to synthesize them in a single indicator, such as the number of infections in HCWs over a period of time, which in turn allows for the estimation of TB infection prevalence and the consequent annual risk of tuberculosis infection (hospital ARTI). Nicas \& Seto [7] simulated the TB infection risk of $\mathrm{HCWs}$ according to their high, medium and low occupational risk and their daily interactions with pulmonary TB patients. Their model assumed that it was possible to estimate LTBI prevalences without assessing some of the variables not directly observable described above.

In Medellín, Colombia, the annual incidence of TB has ranged from $44 \cdot 6$ to $70 \cdot 3$ cases per 100000 habitants [8]. Pulmonary TB accounts for $78 \%$ of the cases. The city's Public Hospital Network (ESE Metrosalud) has 10 hospitals or 'hospital units' (one of which was under restoration while this study was performed), 40 health centres and 2033 employees. ESE Metrosalud manages and oversees most of the TB diagnoses and treatments provided in the city. The effects of social crisis, population dislocation, decay of the healthcare system, and the violent conflict in Colombia have had negative effects on TB control. The Health Care Reform in Colombia, in addition to economic austerity measures, has undermined public health services, including local and national TB control programmes [9]. TB infectiousness falls rapidly after the initiation of effective antituberculous chemotherapy, so undiagnosed patients who are repeatedly admitted to the hospital are likely to be a major source of TB transmission in the city's hospital network.

In Medellín, the control of TB infection in HCWs is a public health priority given its increasing incidence in some parts of the city, the absence of infection control plans and the occurrence of active TB cases in HCWs of some of the hospitals in the public network.

The objective of this study was to simulate LTBI prevalence in HCWs in the city's public network hospitals in order to identify those hospitals with the greater prevalence, leading to priorization of the implementation of TB control plans [10].

The simulation was conducted in nine public hospitals in Medellín, Colombia, incorporating a term for community risk in the model, to reflect intermediate TB incidence countries. In our case, the simulation became the initial phase of the hospital control plan for TB infection in two of the studied hospitals. Afterwards, a prevalence survey was conducted using tuberculin skin tests (TSTs) and methods to measure the production of interferongamma in blood (interferon-gamma release assays; IGRAs). The prevalence data thus obtained was used to validate the simulation.

\section{METHODS}

The frequency of TB infection in HCWs in nine hospitals was simulated to classify the transmission risk (prevalence and hospital ARTI) in each of the hospitals.

The risk of TB infection in HCWs was modelled according to their occupational risk (clinical or administrative), and daily variations in exposure to smear-positive patients who remained untreated for a specific period of time [7].

The model proposed by Nicas \& Seto in 1997 [7] was implemented and modified to obtain a final estimate of the accumulated risk of infection $(R)$. The intensity of exposure $(D)$ and random variability were considered by the expression $R=1-\left(1-\mathrm{e}^{-\boldsymbol{D}}\right)^{n}$, where $n$ denotes the number of exposure periods for HCWs exposed to TB smear-positive patients, expressed in years, and $\mathrm{e}^{-D}$ represents the daily risk of infection $(r)[7,11]$.

\section{The model}

According to Nicas \& Seto [7], the expected number of TB-infected HCWs arises from the accumulated risk of infection, after daily exposure to TB patients during their transmission period and the amount of time they work. Daily infection risk depends on the admission of TB patients during their transmission period and the contact they have with HCWs during the working day [9].

In the model, patient admission was assumed as a random variable with a Poisson distribution. A uniform probability distribution was generated for each susceptible HCW according to whether the individual was assigned to work and if the individual became infected [7]. It was assumed that all patients were equally transmissible. Workers were susceptible at 
the initial time, and re-infection or multiple infections were not considered.

The HCWs were divided into high-, medium- and low-risk occupational groups. The workers' infection risk depends on the risk group they belong to, but the risk is uniform inside each group. The high-risk group have direct contact with patients, and are physically close to them in a full working day. The low-risk group comprises administrative staff with no contact or proximity to patients. The medium-risk group has direct contact with patients for part of the time. One aspect not included by Nicas \& Seto [7] in their model was the non-occupational risk (community ARTI) which is small in low TB incidence countries, but becomes important in high or intermediate TB incidence countries.

The accumulated infection risk, including community ARTI is given by the following expression:

$R=1-\left(1-\left(r_{h}+r_{c}\right)\right)^{n}$,

where $R$ is the accumulated infection risk, $r_{\mathrm{h}}$ is the daily hospital infection risk, $r_{\mathrm{c}}$ is the daily community ARTI, and $n$ is the number of days in the 5-year simulation period [7].

The expected number of infections were obtained by multiplying the accumulated infection risk by the number of workers in each occupational risk group. With these data, the infection prevalence proportion and the ARTI were calculated for each hospital.

\section{Procedures}

To obtain the output variable (the accumulated risk of infection, $R$ ) 4500 simulations were run according to the following procedures:

(a) In order to obtain the number of patients admitted in the hospital by day, a Poisson distribution was generated. The lambda parameter $(n / 365)$, was obtained according to the number of smearpositive patients $(n)$ reported by each hospital between 2009 and 2011.

(b) The cumulative number of patients per day was calculated using 10- and 20-day periods of infectiousness of untreated and partially treated patients. This number was obtained from the original model [7], Colombian and international studies [12, 13] concerning diagnosis delay and summarizes the variability of exposure before diagnosis and during the first TB treatment phase.

(c) The allocation of HCWs in each working day was assumed to follow a uniform distribution, with each employee having the same chance of going to work on a specific day.

(d) The number of infected HCWs each day was determined by generating a uniform distribution. It was considered that the worker was infected if the value obtained from the probability distribution was less than or equal to a calculated daily risk of infection in the hospital. HCWs who were considered infected were subtracted from the susceptible group to avoid doublecounting infections.

(e) To run the simulation, the initial values of the daily risk of infection in hospitals were obtained from a meta-analytical study reporting the summary measures of the annual incidence (\%) of LTBIs in HCWs for countries of intermediate incidence [3]. In our simulation, we used the confidence intervals for this incidence in the following way: the upper confidence interval for the HCWs in the high-risk occupational group $(10 \cdot 31 \%)$, the interval point estimation for the medium-risk group (6.87\%), and the lower confidence interval for the low-risk group (3.43\%) [3]. These data were converted to a daily risk for each risk group (e.g. 10·31/100/365).

$(f)$ The estimated $1 \cdot 2 \%$ community ARTI for Medellín was obtained from data published by the regional health authority (Dirección de Salud de Antioquia) [8]. We divided the pulmonary $\mathrm{TB}$ rate by 50 given that according to the Styblo rule a community ARTI of $1 \%$ in underdeveloped countries is equivalent to nearly 50 new smear-positive cases $/ 100000$ population $[4,14]$. We then converted it to daily risk.

( $g$ ) Once $R$ was calculated, we multiplied it by the size of each group of HCWs to estimate the expected number of infections in each group. Using this result, the LTBI prevalence ( $p$ ) was estimated for each of the nine hospitals.

(h) Finally, using the prevalence of LTBI $(p)$, the ARTI for each hospital was calculated using the expression $1-(1-p)^{1 / T}$, where $T$ denotes the median years that HCWs were employed at each hospital (Table 1) [15].

\section{Sensitivity analysis and validation}

The sensitivity analysis examined whether variations in the length of time that patients were infectious, and the daily risk of infection could have significant impacts on 
Table 1. Patients diagnosed with TB (smear-positive), number of healthcare workers ( $H C W s)$ at high, medium and low occupational risk, and median of years that HCWs were employed in nine hospitals in the Public Hospital Network of Medellin, Colombia

\begin{tabular}{lllllll}
\hline \hline \multirow{2}{*}{$\begin{array}{l}\text { Hospital } \\
\text { TB-diagnosed } \\
\text { patients (mean) }\end{array}$} & \multicolumn{2}{l}{ Number of HCWs } & & $\begin{array}{l}\text { Years HCWs } \\
\text { employed (median) }\end{array}$ \\
\cline { 3 - 6 } & High risk* & Medium risk $\dagger$ & Low risk $*$ & Total & 127 & $14 \cdot 1$ \\
BA & 106 & 85 & 16 & 26 & 103 & $16 \cdot 4$ \\
SCR & 79 & 68 & 12 & 23 & 145 & $10 \cdot 6$ \\
MA & 30 & 106 & 12 & 27 & 150 & $14 \cdot 8$ \\
BE & 27 & 100 & 16 & 34 & 114 & $14 \cdot 0$ \\
CA & 37 & 74 & 19 & 21 & 146 & $14 \cdot 5$ \\
SJ & 22 & 104 & 11 & 31 & 102 & $10 \cdot 8$ \\
DO & 25 & 65 & 15 & 22 & 93 & $18 \cdot 3$ \\
SC & 19 & 50 & 12 & 31 & 102 & $15 \cdot 4$ \\
SAP & 5 & 56 & 8 & 38 & 1082 & $14 \cdot 3$ \\
Total & 350 & 708 & 121 & 253 & \\
\hline \hline
\end{tabular}

$*$ Daily risk of infection $=0.0003153425$.

$\dagger$ Daily risk of infection $=0.0002210959$.

$\ddagger$ Daily risk of infection $=0.000126849$.

the simulation results. Four different scenarios were assessed, using 5-, 10-, 15- and 20-day periods of infectiousness of untreated and partially treated patients and the annual incidence of LTBI in high-incidence countries [high-risk (14.05\%), medium-risk $(8 \cdot 40 \%)$, and low-risk $(2 \cdot 75 \%)$ occupational groups] [3].

The external validity of the simulation was assessed by comparing the prevalence obtained in the model with that one obtained from the LTBI prevalence survey in HCWs in two hospitals (BE and MA) $(n=408)$. All HCWs reside in a country with universal bacille Calmette-Guérin (BCG) vaccine coverage at birth [16]. The prevalence survey used TSTs [5 TU (0.1) ml of Tubersol ${ }^{\circledR}$ PPD, Sanofi Pasteur Ltd, Canada] with a cut-off point for positivity of $10 \mathrm{~mm}$, and IGRAs [QuantiFERON-TB Gold In-Tube (QFT); Cellestis/Qiagen, Australia], whose results were considered positive when the test values were $\geqslant 0.35 \mathrm{IU} /$ $\mathrm{ml}$ according to the manufacturer's instructions. The number of HCWs undergoing laboratory tests was higher than those in the simulation. This was due to the fact that the total number of HCWs for the simulation was taken from the institutional personnel registry between 2009 and 2011, while the number of HCWs for the prevalence survey at BE and MA hospitals was based on a census of personnel conducted on site between 2012 and 2013. It was found that the actual number of HCWs on site was higher than that of the registry because it included personnel working under contract with other different employment agencies.
The simulation was run with an MS Excel (Microsoft Corporation, USA) macro. The algorithm developed for the simulation is available from the authors upon request. The simulation was verified by running the same model with a tool developed in Powersim Studio 8 Academic $^{\circledR}$ (Powersim Software AS 2013, Norway).

\section{Ethical statement}

The authors assert that all procedures contributing to this work comply with the ethical standards of the relevant national and institutional committees (Bioethics Review Committee of The National School of Public Health, University of Antioquia: C13255-161040 and the Management Committee at ESE Metrosalud) on human experimentation and with the Helsinki Declaration of 1975, as revised in 2008.

\section{RESULTS}

The number of HCWs in each hospital was between 100 and 150, with most working in patient care, $65 \cdot 4 \%$ were classified as being at high, $11 \cdot 2 \%$ at medium and $23.4 \%$ at low occupational risk. The median number of years employed was 14.3 (inter-quartile range 4.2-21.2). Two of the hospitals (BA and SCR), reported the highest average of diagnosed patients with smear-positive pulmonary TB (Table 1).

Table 2 summarizes the results of the 5-year simulation period for the three groups of workers exposed 
to 10- and 20-day TB patients in their contagious period at the nine hospitals under study.

In the model, increased numbers of high-risk workers and more days of exposure to smear-positive TB patients increased the average number of HCWs infected as well as the overall LTBI prevalence and the hospital ARTI (Table 2).

The average number of LTBIs was related to exposure time. For 10 days of exposure, the average number of infections ranged from 4 to 73 ; and for 20 days, it ranged from 8 to 100 . Full-time clinical workers (high-risk occupational group) showed the highest number of infections.

The average number of infected HCWs in each hospital was variable, with higher numbers in BA and SCR hospitals and lower numbers in SC and SAP hospitals, for both exposure periods (Table 2).

The prevalence of simulated LTBIs ranged from $3.9 \%$ to $57.5 \%$ for 10 days of exposure, and from $7 \cdot 8 \%$ to $78.7 . \%$ for 20 days of exposure. For a 10-day exposure period, the prevalence of infection in BA and SCR hospitals was $>25 \%$. In five hospitals the prevalence ranged between $15 \%$ and $25 \%$ and was $<15 \%$ in SC and SAP hospitals. For 20 days of exposure, the prevalence was $>25 \%$ in all hospitals except for SAP (Table 2).

The simulated hospital ARTI was higher than the community ARTI in seven (10-day exposure period) and in eight out of the nine hospitals (20-day exposure period). The highest hospital ARTI was $10 \cdot 4 \%$. The simulated prevalence data were used as the cut-off point to classify hospitals into three categories of risk level: very high priority (BA and SCR hospitals, which are located in areas of the city with high $\mathrm{TB}$ incidence); high priority (CA, MA, BE, SJ, DO, SC hospitals); and low priority (SAP hospital).

The sensitivity analysis obtained similar results running the model with the same parameter values (data not shown). Figure 1 shows the change over time in the total number of LTBIs in HCWs over the hypothetical 5-year simulation period, for intermediate and high daily infection risks, and for 5, 10, 15 and 20 days of exposure. The sensitivity analysis showed similar results despite the change in daily risk of infection.

In the analysis of the prevalence survey, we used concordant positive QTF and TST (QTF+/TST+) tests as the numerator. The LTBI prevalence results obtained in the survey were similar to those of the simulation. At MA hospital, the LTBI survey prevalence was $41 \%$ [90/220, 95\% confidence interval (CI) 34.3-47.7] and at BE hospital 44\% (82/188, 95\% CI 36.4-51.0). In the simulation, the LTBI prevalence was $40 \cdot 7 \%(95 \%$ CI 32.3-49.0) at MA and 36\% at BE (95\% CI 27.9-44.0) hospitals for the 20-day exposure period.

\section{DISCUSSION}

The starting point of the present study was the model proposed by Nicas \& Seto in the 1990s [7, 11]. They simulated infection risk according to occupational risk. This study extended the model by including community risk alongside occupational risk, and by applying the simulation to nine hospitals in an intermediate TB incidence city. Using the simulated LTBI prevalence, we classified the hospitals by levels of risk prior to the realization of a prevalence LTBI survey using TSTs and IGRAs (QTF).

Whenever periodic prevalence surveys are feasible, the simulation allows for the identification of hospitals where a survey should be conducted, according to the higher simulated LTBI prevalences. Control measures can be then targeted to high-risk occupational groups and TB infection control goals can be settled using critical indicators of exposure reduction of $\mathrm{HCWs}$ (i.e. time between diagnosis and treatment, and the isolation of patients with pulmonary TB). Moreover, the simulation allows for the estimation of sample sizes for prevalence surveys when it is not feasible to study all HCWs.

The simulation also allows for the analysis of the relationship between patient admission and the probability of contact with $\mathrm{HCWs}$, considering the inherent variability of the phenomenon. Although the mean number of smear-positive diagnosed patients is an important factor for infection risk, other factors such as the length of the HCWs' exposure to contagious patients, the HCWs' occupational risk category (clinical vs. administrative), their period of employment, and the duration of the working day can modify hospital ARTI and LTBI prevalence. The advantage of the Nicas-Seto model is that it summarizes the cumulative dose of infection in a single expression that preserves the effect of time and the particular risk of infection associated with those factors described above. Other authors have assessed the risk of TB infection and included several other factors that influence the degree of exposure, such as the frequency of transmission by patients with infective droplet nuclei ('quanta of infection'), the volume of 


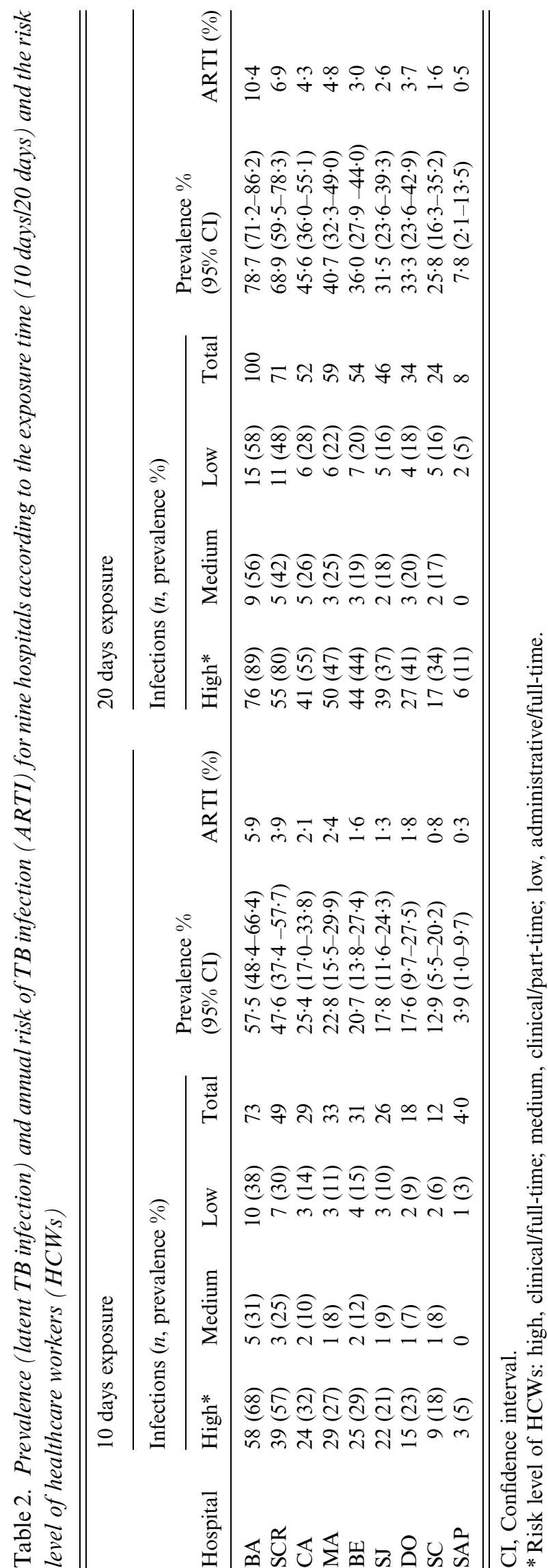

air inhaled by the subjects, and the ventilation of the environment $[17,18]$. The disadvantage of the latter methods is that the parameter values required in these cases demand complex procedures such as measurement of room ventilation not usually available to the TB infection control teams in hospitals of low-income countries.

All mathematical epidemiological models are limited by the assumptions made. Several assumptions were made in our model which may limit the validity of the results. First, all patients were assumed to be of equal infectiousness, which does not reflect the variability in clinical reality. This variability in contagiousness has been studied by Fennely et al. [19] and Williams et al. [20] using experimental methods, these authors found that the amount of aerosol production varies from patient to patient with both positive TB smears and cultures. There were no data available to assess different levels of patient infectiousness since sputum culture is not performed routinely for all patients. We ran the model using a variety of HCWs' daily risk and different lengths of exposure to contagious patients (10 and 20 days). The sensitivity analysis showed consistency with the simulation results, when the initial values of occupational risk were modified and when the transmissibility time of TB patients was changed. Given that the same assumption was made for all hospitals studied, the assumption regarding equal infectiousness of patients resulted in a systematic limitation which did not affect one hospital more than another.

A further assumption that was made to simplify the model was that only smear-positive patients were considered as a source of infection. Given that some transmission does occur from smear-negative, culturepositive patients, this would result in an underestimate of the overall risk to HCWs. However, given that the proportion of TB transmission attributable to smearnegative patients in thought to be small $[21,22]$ this assumption would likely only result in a small underestimate of overall risk, and again would be similar for each hospital.

Finally, we made the assumption that all of the HCWs were initially susceptible, within two levels of exposure corresponding to $\mathrm{HCWs}$ in clinical vs. administrative activities. The homogeneity of susceptibilty within each of those levels of risk could underestimate the simulated prevalence.

Overall, in order to improve this type of simulation model, it is necessary to include the heterogeneity of susceptibility of HCWs [23, 24], the variability of 

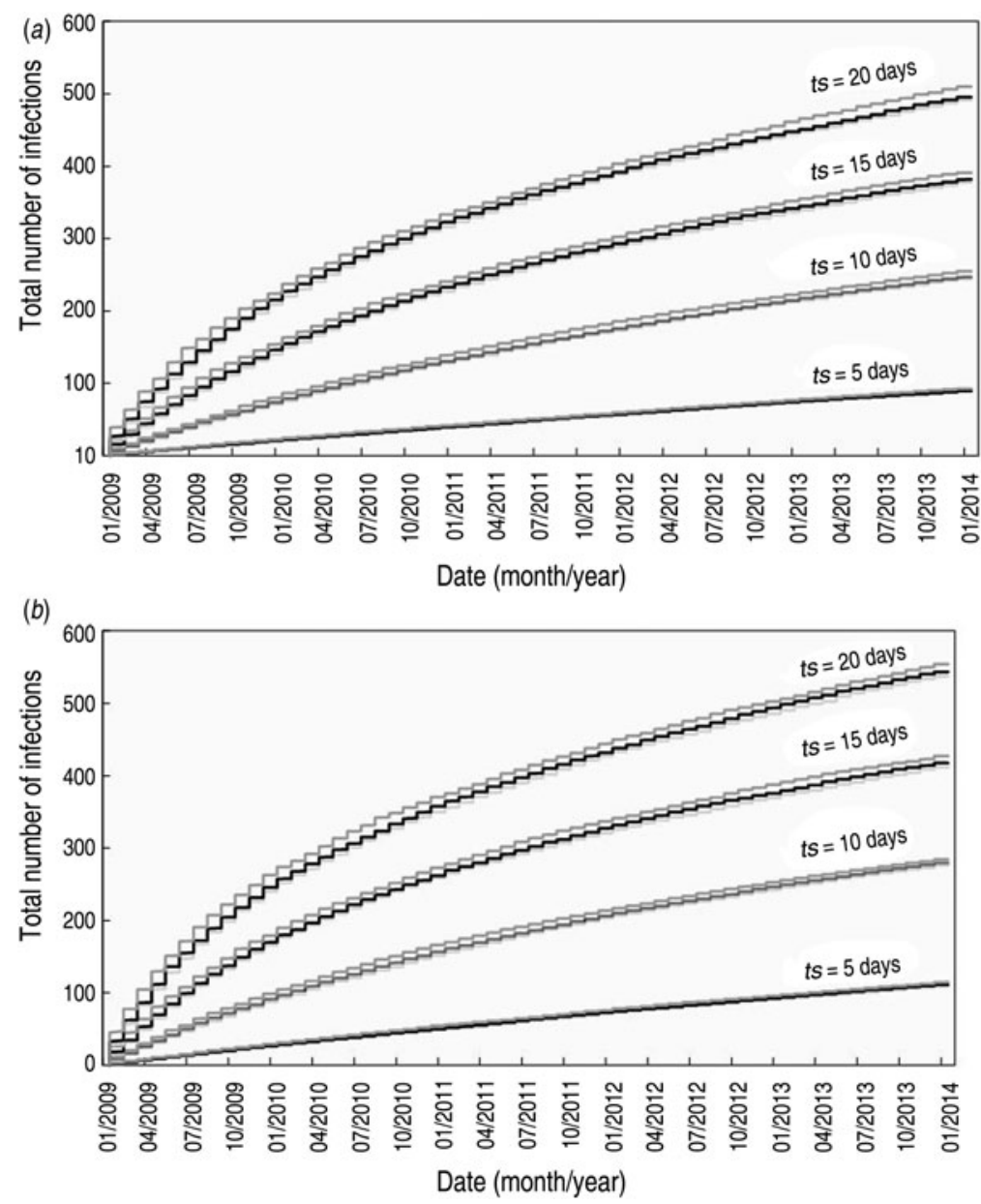

Fig. 1. Sensitivity analysis: total number of infected HCWs over a hypothetical 5-year simulation period in nine hospitals during periods of infectiousness of untreated and partially treated patients: 5, 10, 15 and 20 days. Daily infection risks of (a) intermediate TB incidence countries, $(b)$ high TB incidence countries.

exposure and the occurrence of multiple exposures such as the exposure observed with diagnosed patients and other non-observed exposures with asymptomatic or undetected symptomatic patients [25].

Regarding the validation of the simulation, an adequate adjustment was found between the simulated and prevalence surveys in the two hospitals with available data. We used concordant positive QTF and TST $(\mathrm{QTF}+\mathrm{TST}+)$ tests as the numerator for LTBI prevalence given that little is known about the meaning of discordant combinations as reported by Nienhaus et al. [26]. In Colombia, for example, BCG is administered universally to all the population at birth, which may confound TST results with false positives. Moreover, both QTF and TST are imperfect tests in the absence of a gold standard for LTBIs, and using the tests together may increase the sensitivity [27]. A study by Mirtskhulava et al. [28] also used concordant positives (QTF+/TST+) and found a 50\% LTBI prevalence in a high-incidence country, which is similar to that found in our survey.

A contribution made by our study is the inclusion of community ARTIs to estimate the number of LTBIs, a parameter not included in the Nicas-Seto simulation because the US population has a low community risk of LTBI. Following the authors, the annual risk of infection in the city was estimated so as to reflect the potential of community exposure of the HCWs in a context of intermediate TB incidence countries. These data have limitations: the study was performed using averages that do not reflect the heterogeneity of the risk of infection within the city. Since the same equal number was given to community ARTIs for all the city, we may be underestimating the risk for the place of residence of some of the HCWs. Further, it was not possible to assess the real exposure time of the HCWs to patients attending each hospital, due to the lack of hospital control plans, and 
therefore, lack of follow-up to the occupational history of HCWs.

Another contribution of this study was to estimate the ARTIs of each hospital using the median of years spent at work for their HCWs. However, the numbers may be underestimated because the data on the exposure of HCWs in the different hospitals where they worked is not disaggregated.

The prevalence and ARTI values simulated in this study are consistent with those published using TST surveys in Latin American countries with intermediate incidence rates of TB [3, 29]. In 2007, Joshi et al. [30] reported a LTBI prevalence between $33 \%$ and $79 \%$ in HCWs in low- and middle-income countries, and reported an ARTI between $0 \cdot 5 \%$ and $14.3 \%$. The simulation results presented herein match these values. In 2005, Roth et al. [29] published a LTBI prevalence of $63 \cdot 1 \%$ for hospitals in Brazil (a country with an intermediate TB incidence similar to Colombia), reaffirming the similarities.

This study highlights the importance of TB infection control measures for reducing the transmission of TB to HCWs. A variety of measures may be employed, such as triage and isolation to reduce exposure time, and cough etiquette to reduce production of infectious aerosols [10, 31].

Simulation was run with a MS Excel spreadsheet suitable for use by regional managers and staff members who develop infection control programmes for HCWs. Public health authorities and hospital managers may use simulation outputs obtained by this model, modify its set of parameters and guide the activities of infection control in all hospitals, particularly those classified as high priority.

The simulation proposed here could be a helpful tool for estimating TB infection risk in HCWs when it is not feasible to conduct prevalence surveys using laboratory tests. Results will also be helpful for the classification of the risk level in hospitals and promote measures for infection control.

\section{ACKNOWLEDGEMENTS}

The authors thank the funding agencies and the directors and staff of ESE Metrosalud. In particular, we thank Emilia Ochoa, Rita Almanza, Fernando Montes, Clara Trujillo, Verónica Lopera, Gladys Ariza, Rubiela Álvarez, Ana E. Correa, Gloria Uribe, Nilton E. Montoya, Jaminson Ricardo, Angélica Pérez, Jorge A. Mejía and Leonardo
Uribe. We thank the anonymous reviewers for their insightful comments and suggestions.

This work was funded by University of Antioquia (The Sustainability Strategy 2013-2014; Bank Programs and Projects of the University Extension code 15313313), ESE Metrosalud, Secretariat of Health of Medellín (Agreement No. 4600042433) and by the Administrative Department of Science and Innovation of Colombia (COLCIENCIAS code: 111556934195)

\section{DECLARATION OF INTEREST}

None.

\section{REFERENCES}

1. WHO. WHO policy on TB infection control in healthcare facilities, congregate settings and households. WHO/HTM/TB/2009·419. Geneva, Switzerland: WHO, 2009.

2. Dharmadhikari AS, et al. Surgical face masks worn by patients with multidrug-resistant tuberculosis: impact on infectivity of air on a hospital ward. American Journal of Respiratory and Critical Care Medicine 2012; 185: 1104-1109.

3. Baussano I, et al. Tuberculosis among health care workers. Emerging Infectious Diseases 2011; 17: 488-494.

4. Sepkowitz KA. Tuberculosis and the health care worker: a historical perspective. Annals of Internal Medicine 1994; 120: 71-79.

5. Claassens MM, et al. Tuberculosis in healthcare workers and infection control measures at primary healthcare facilities in South Africa. PLOS ONE 2013; 8: e76272.

6. Nardell EA. Catching droplet nuclei: toward a better understanding of tuberculosis transmission. American Journal of Respiratory and Critical Care Medicine 2004; 169: 553-554.

7. Nicas M, Seto E. A simulation model for occupational tuberculosis transmission. Risk Analysis 1997; 17: 609-616.

8. Anon. Colombia. Government of Antioquia. World Day of the fight against tuberculosis. Bulletin information for action - BIA-Medellin, March 2012 (http://www. dssa.gov.co/index.php/documentos-de-interes/vigilanciaepidemiologica/bias/2012/779-marzo-2012/file). Accessed 14 December 2013.

9. Arbelaez MP, et al. Tuberculosis control and managed competition in Colombia. International Journal of Health Planning and Management 2004; 19 (Suppl. 1): S25-43.

10. Jensen PA, et al. Guidelines for preventing the transmission of Mycobacterium tuberculosis in health-care settings, 2005. Morbidity and Mortality Weekly Report Recommendations and Reports 2005; 54: 1-141. 
11. Nicas M. An analytical framework for relating dose, risk, and incidence: an application to occupational tuberculosis infection. Risk Analysis 1996; 16: 527-538.

12. Sreeramareddy CT, et al. Time delays in diagnosis of pulmonary tuberculosis: a systematic review of literature. BMC Infectious Diseases 2009; 9: 91.

13. Caceres-Manrique Fde M, Orozco-Vargas LC. Delayed diagnosis of pulmonary tuberculosis in a particular part of Colombia. Revista de Salud Publica (Bogota, Colombia) 2008; 10: 94-104.

14. Styblo K. The relationship between the risk of tuberculous infection and the risk of developing infectious tuberculosis. Bulletin of the International Union against Tuberculosis and Lung Disease 1985; 60: 117-199.

15. Sutton PM, et al. Is a tuberculosis exposure a tuberculosis exposure if no one is infected? Infection Control and Hospital Epidemiology 1999; 20: 92-97.

16. Zwerling A, et al. The BCG World Atlas: a database of global BCG vaccination policies and practices. PLoS Medicine 2011; 8: e1001012.

17. Nazaroff WW, Nicas M, Miller SL. Framework for evaluating measures to control nosocomial tuberculosis transmission. Indoor Air 1998; 8: 205-218.

18. Nardell EA, et al. Airborne infection. Theoretical limits of protection achievable by building ventilation. American Review of Respiratory Disease 1991; 144: 302-306.

19. Fennelly KP, et al. Variability of infectious aerosols produced during coughing by patients with pulmonary tuberculosis. American Journal of Respiratory and Critical Care Medicine 2012; 186: 450-457.

20. Williams CM, et al. Face mask sampling for the detection of Mycobacterium tuberculosis in expelled aerosols. PLoS ONE 2014; 9: e104921.

21. Behr MA, et al. Transmission of Mycobacterium tuberculosis from patients smear-negative for acid-fast bacilli. Lancet 1999; 353: 444 449.
22. Tostmann A, et al. Tuberculosis transmission by patients with smear-negative pulmonary tuberculosis in a large cohort in the Netherlands. Clinical Infectious Diseases 2008; 47: 1135-1142.

23. Woolhouse ME, et al. Heterogeneities in the transmission of infectious agents: implications for the design of control programs. Proceedings of the National Academy of Sciences USA 1997; 94: 338-342.

24. Lopes JS, et al. Interpreting measures of tuberculosis transmission: a case study on the Portuguese population. BMC Infectious Diseases 2014; 14: 340.

25. Payet $\mathbf{C}$, et al. A statistical model to assess the risk of communicable diseases associated with multiple exposures in healthcare settings. BMC Medical Research Methodology 2013; 13: 26.

26. Nienhaus A, et al. IFN-gamma release assay versus tuberculin skin test for monitoring TB infection in healthcare workers. Expert Review of Anti-infective Therapy 2013; 11: 37-48.

27. Denkinger CM, Dheda K, Pai M. Guidelines on interferon-gamma release assays for tuberculosis infection: concordance, discordance or confusion? Clinical Microbiology and Infection 2011; 17: 806-814.

28. Mirtskhulava V, et al. Prevalence and risk factors for latent tuberculosis infection among health care workers in Georgia. International Journal of Tuberculosis and Lung Disease 2008; 12: 513-519.

29. Roth VR, et al. A multicenter evaluation of tuberculin skin test positivity and conversion among health care workers in Brazilian hospitals. International Journal of Tuberculosis and Lung Disease 2005; 9: 1335-1342.

30. Joshi R, et al. Tuberculosis among health-care workers in low- and middle-income countries: a systematic review. PLoS Medicine 2006; 3: e494.

31. Jesudas CD, Thangakunam B. Tuberculosis risk in health care workers. Indian Journal of Chest Diseases \& Allied Sciences 2013; 55: 149-154. 